J. Clin. Chem. Clin. Biochem.

Vol. 23, 1985, pp. 293-301

\title{
Mineral- und Spurenelemente im menschlichen Urin
}

\author{
Von P. Schramel, G. Lill und S. Hasse \\ Gesellschaft für Strahlen- und Umweltforschung $m b H$, \\ Institut für Angewandte Physik, Physikalisch-Technische Abteilung, Oberschleißheim
}

(Eingegangen am 22. Oktober 1984/4. Februar 1985)

Zusammenfassung: Mittels ICP (Inductively Coupled Plasma)- und DCP (Direct Current Plasma)-Emissionsspektroskopie wurden in etwa hundert 24-Stunden-Sammelurin-Proben eines Referenzkollektivs (Alter zwischen 20 und 50 Jahre) die Konzentrationen der Elemente $\mathrm{Al}, \mathrm{B}, \mathrm{Ca}, \mathrm{Fe}, \mathrm{K}, \mathrm{Li}, \mathrm{Mg}, \mathrm{Na}, \mathrm{P}$ und $\mathrm{Zn}$ untersucht. Die dabei erhaltenen Werte können auf Grund der großen Probenzahl als „,Referenzwerte“ angesehen werden. Es wurden die Mittelwerte sowie der Bereich, bezogen auf Volumen (Konzentration) als auch bezogen auf 24 Stunden (Ausscheidung), angegeben. Für den Bereich der Arbeitsmedizin werden solche physiologische Referenzwerte für die Elemente $\mathrm{Ba}, \mathrm{Sr}$ und $\mathrm{Ti}$ und einige Werte für Belastungsfälle gezeigt. Mittels voltammetrischer Bestimmungsmethoden werden zusätzlich Durchschnittswerte für $\mathrm{Cd}, \mathrm{Co}, \mathrm{Ni}$ und $\mathrm{Pb}$ angegeben.

\section{Mineral and trace elements in human urine}

Summary: Mineral and trace elements ( $\mathrm{Al}, \mathrm{B}, \mathrm{Ca}, \mathrm{Cu}, \mathrm{Fe}, \mathrm{K}, \mathrm{Li}, \mathrm{Mg}, \mathrm{Na}, \mathrm{P}$ and $\mathrm{Zn}$ ) in 24 hour human urine samples from approximately 100 healthy persons (age between 20 and 50 years) were analysed by ICP (Inductively Coupled Plasma) and DCP (Direct Current Plasma) emission spectroscopy. The obtained values can be taken as "reference values" owing to the large number of individual samples. Mean values and the range of substance concentrations and substance excretion per 24 hours are given. Physiological reference values are reported for $\mathrm{Ba}, \mathrm{Sr}$ and $\mathrm{Ti}$, which are of particular interest in the field of occupational medicine; examples of increases in these three elements as a result of exposure at the work place are also given. Additionally some averarage values for $\mathrm{Cd}, \mathrm{Co}, \mathrm{Ni}$ and $\mathrm{Pb}$ have been measured by a voltammetric technique.

\section{Einführung}

Für den Bereich der Mineral- und Spurenelementanalytik in menschlichen Urinproben - speziell für die Klinische Chemie und Arbeitsmedizin - bietet die ICP (Inductively Coupled Plasma)- bzw. DCP (Direct Current Plasma)-Emissionsspektroskopie eine ganze Reihe von Vorteilen im Vergleich zu den konventionellen Techniken der Flammen-Emission und -Absorption. Sie liegen einerseits in der Möglichkeit, mehr relevante Elemente zu bestimmen und andererseits, bei Verwendung eines Simultanspektrometers, in der Möglichkeit der gleichzeitigen Bestimmung von medizinisch relevanten Elementen bei einem relativ niedrigen Probenverbrauch $(5-10 \mathrm{ml}$ für die hier behandelten Elemente) sowie in der kurzen Analysendauer (etwa $5 \mathrm{~min} /$ Probe). Darüber hinaus ist die Plasma-Emissionsspektroskopie im Gegensatz zur Flammen-Emissions- und Absorptionsspektrometrie eine relativ störungsarme Methode - vor allem hinsichtlich von chemischen Matrixeinflüssen - so daß derartige Bestimmungen auch mit einem hohen Grad an Richtigkeit und Reproduzierbarkeit durchgeführt werden können. Nicht zuletzt ist das Fehlen jeglicher Probenvor- oder -aufbereitung auch ein entscheidender Faktor für die praktische Anwendung im Labor. Damit treten auch keinerlei Probleme mit Blindwerten aus Reagentien o. ä. auf. 
Die bei diesen Anregungsverfahren für die Emissionsspektroskopie bekannten Störungen wie Linien- und Bandenkoinzidenzen, Untergrundbeeinflussung, Linienumkehr und Zerstäubereffekte spielen bei den hier betrachteten Elementen entweder keine Rolle oder sind auf Grund der relativ einheitlichen Matrix beherrschbar.

Aus diesen Gründen soll hier die Anwendung dieser analytischen Verfahren für die Bestimmung der Elemente $\mathrm{Al}, \mathrm{Ca}, \mathrm{Cu}, \mathrm{Fe}, \mathrm{K}, \mathrm{Li}, \mathrm{Mg}, \mathrm{Na}, \mathrm{P}, \mathrm{Zn}$ sowie die von $\mathrm{Ba}, \mathrm{Sr}$ und $\mathrm{Ti}$ vorgestellt werden. $\mathrm{Da}$ es für aussagekräftige Diagnosen über die Urinausscheidung im Bereich der Klinischen Chemie und dem der Arbeitsmedizin meist notwendig ist, 24-h-Sammelurin-Proben zu verwenden, wurden für diese Untersuchungen etwa 100 Sammelurin-Proben eines Normalkollektivs (Männer und Frauen, Alter zwischen 20-50 Jahre) analysiert. Auf Grund der großen Probenzahl können die dabei erhaltenen Werte als „Referenzwerte" angesehen werden.

Besonders für den Bereich der Arbeitsmedizin (hier speziell hinsichtlich möglicher Arbeitsplatzbelastungen) wurden die Elemente $\mathrm{Ba}, \mathrm{Sr}$ und $\mathrm{Ti}$ miteinbezogen, da es für diese Elemente keine andere vergleichbare analytische Methode gibt, die es gestattet, den physiologischen und den arbeitsmedizinisch relevanten Konzentrationsbereich im Urin zu erfassen. Aus dem gleichen Grund werden mittels voltammetrischer Technik gemessene Werte für die Elemente $\mathrm{Cd}, \mathrm{Co}$, $\mathrm{Ni}$ und $\mathrm{Pb}$ angegeben. Das Element Li gewinnt auf Grund seiner vermuteten Essentialität immer mehr an Bedeutung (1).

Das Interesse der Klinischen Chemie und der Arbeitsmedizin an der Untersuchung von Mineral- und Spurenelementen im Urin zur Diagnose oder zur Therapiekontrolle ist unbestritten (2-4), und in jedem Fall liefert die Plasma-Emissionsspektroskopie gleichzeitig eine Vielzahl von möglicherweise wichtigen Informationen, die mit keiner anderen Technik in der Routine zu erhalten sind.

\section{Material und Methoden \\ Probensammlung und -vorbereitung}

An etwa 100 Personen eines Referenzkollektivs - ohne wissentliche Erkrankung oder Medikamenteneinnahme - im Alter zwischen 20-50 Jahren (etwa gleiche Anzahl von männlichen und weiblichen Probanden) wurden vorher mit verdünnter $\mathrm{HNO}_{3}$ gereinigte Polyethylen-Flaschen zur Urinsammlung ausgegeben. Die Sammlung wurde wegen der Praktikabilität jeweils an einem Wochenende durchgeführt. Unmittelbar nach der Abgabe wurde die totale Urinmenge bestimmt. Unter ständigem Rühren wurde daraus ein Aliquot von $100 \mathrm{ml}$ entnommen und mit $10 \mathrm{ml}$ hochreiner $\mathrm{HNO}_{3}$ (sub boiling destilliert) angesäuert und bis zur Analyse tiefgefroren $\left(-25^{\circ} \mathrm{C}\right)$. Vor der Analyse wurden die Proben wieder aufgetaut und für die Elemente $\mathrm{Al}$, $\mathrm{B}, \mathrm{Ba}, \mathrm{Cu}, \mathrm{Fe}, \mathrm{Li}, \mathrm{Mn}, \mathrm{Ti}$ und $\mathrm{Zn}$ ohne weitere Behandlung der Messung zugeführt. $\mathrm{Sr}$ wurde in einer 1:10-Verdünnung gemessen, für die Elemente $\mathrm{Ca}, \mathrm{K}, \mathrm{Mg}, \mathrm{Na}$ und $\mathrm{P}$ wurde eine 1:50-Verdünnung hergestellt. Auch diese Verdünnungen wurden jeweils mit $\mathrm{HNO}_{3}$ im Verhältnis 1:10 angesäuert. Es sollte hier hervorgehoben werden, daß $\$$ diese Verdünnungen von den eingestellten Gerätekenngrößen abhängig sịnd. Bei Optimierung dieser auf Urinproben können alle Elemente auch in der konzentrierten Probe gemessen werden. Für die voltammetrische Bestimmung von $\mathrm{Cd}, \mathrm{Co}, \mathrm{Ni}$ und $\mathrm{Pb}$ ist eine vollständige Zerstörung der organischen Bestandteile notwendig, so $\mathrm{da} \beta$ hier mit Veraschung der Ưrinprobe gearbeitet werden muß.

Dafür wurde eine modifizierte Druckveraschung (5) mit $\mathrm{HNO}_{3}$ durchgeführt. Dazu wurden $5 \mathrm{ml}$ Urin in ein Quarzveraschungsglas pipettiert und gefriergetrocknet (Produkttemperatur etwa $10^{\circ} \mathrm{C}$ ). $\mathrm{Zu}$ dem trockenen Material wurden $4 \mathrm{ml} \mathrm{HNO}_{3}, 0,5 \mathrm{ml}$ $\mathrm{HClO}_{4}$ und $0,1 \mathrm{ml} \mathrm{H}_{2} \mathrm{SO}_{4}$ gegeben, das Quarzglas in die Druckveraschungsapparatur eingesetzt und 12 Stunden bei $140^{\circ} \mathrm{C}$ verascht. Die Aufschlußlösung wurde in einen Quarztiegel umgefüllt und auf einer Heizplatte $\left(200^{\circ} \mathrm{C}\right)$ zum Trocknen eingedampft; anschließend wurden $4 \times$ je $0.5 \mathrm{ml} \mathrm{HClO}_{4}$ einpipettiert und jeweils wieder zum Trocknen eingedampft, um das organische Material vollständig zu zerstören. Der Rückstand wurde schließlich mit $20 \mu \mathrm{HClO}_{4}$ und $10 \mathrm{ml} \mathrm{H}_{2} \mathrm{O}$ aufgenommen, dies stellte dann die Probenlösung dar $(\mathrm{pH}=2,0)(6)$. Die Blindwerte der gesamten Prozedur liegen für Cd und Co bei $0,2 \mathrm{ng}$ / Aufschluß, für Ni bei $5 \mathrm{ng} /$ Aufschluß und für $\mathrm{Pb}$ bei $2 \mathrm{ng} /$ Aufschluß.

\section{Analytische Methoden}

ICP-Emissionsspektroskopie

\section{a) Sequenz-Spektrometer}

Die Elemente $\mathrm{Ba}, \mathrm{Sr}$ und $\mathrm{Ti}$ wurden, da diese Elemente nicht im Simultanspektrometer installiert waren, mit.Hilfe des Sèquenzspektrometers JY 38 (Instruments S.A.) gemessen $(7,8)$. In Tabelle 1 sind die zur Messung verwendeten Wellenlängen angegeben.

Tab.1. Verwendete Wellenlängen am ICP-Sequenzspektrometer.

\begin{tabular}{ll}
\hline Element & Wellenlänge (nm) \\
\hline $\mathrm{Ba}$ & 455,40 \\
$\mathrm{Sr}$ & 407,77 \\
$\mathrm{Ti}$ & 334,94 \\
\hline
\end{tabular}

b) Simultan-Spektrometer

Zur Bestimmung der Elemente $\mathrm{B}, \mathrm{Ca}, \mathrm{Cu}, \mathrm{Fe}, \mathrm{Mg}, \mathrm{P}$ und $\mathrm{Zn}$ stand ein Simultanspektrometer JY 48 (Instruments S.A.) zur Verfügung $(9,10)$. Tabelle 2 zeigt die zur Messung verwendeten Wellenlängen.

In beiden Fällen (Sequenz- und Simultangerät) wurde mit adäquater Korrektur des Untergrunds gearbeitet. Als Zerstäuber wurde ein Ringspaltzerstäuber mit Pt-Ir-Kapillare (Instruments S.A.) mit wassergekühlter Zerstäuberkammer (11) eingèsetzt. Durch die Verwendung einer peristaltischen Pumpe in der Pro: benzuleitung ist der Probenverbrauch auf etwa $0,9 \mathrm{ml} / \mathrm{min}$ reduziert (12). Es wurde in allen Fällen mit einem $\mathrm{Ar} / \mathrm{H}_{2}$ Gemisch (Volumenanteil 0,935/0,065) (13) für alle Gase (Kühl-, Plasma - und Aerosolträgergạs) gearbeitet. Darüber hinaus waren für alle Gasströmungen elektronische Mass Flow-Regler (11) zur besseren Stabilität der Emissionssignale eingebaut. Die Zeitdauer für eine Analyse ( 3 Wiederholungen) beträgt am Sequenzgerät etwa 1,5 min/Probe und Element (Integrationszeit 1 s) und am Simultanspektrometer etwa $4 \mathrm{~min} /$ Probe (Integrationszeit $10 \mathrm{~s}$ ). 
Tab. 2. Verwendete Wellenlängen am ICP-Simultanspektrometer.

\begin{tabular}{ll}
\hline Element & Wellenlänge (nm) \\
\hline B & 249,67 \\
$\mathrm{Ca}$ & 393,37 \\
$\mathrm{Cu}$ & 324,75 \\
$\mathrm{Fe}$ & 259,94 \\
$\mathrm{Mg}$ & 279,55 \\
$\mathrm{P}$ & 253,56 \\
$\mathrm{Zn}$ & 213,85 \\
\hline
\end{tabular}

\section{DCP-Emissionsspektroskopie}

Für die quantitative Bestimmung der Elemente $\mathrm{Al}, \mathrm{K}, \mathrm{Li}$ und $\mathrm{Na}$ wurde ein DCP-Spektrometer Spectra Span VI (Beckman) eingesetzt (14). Dieses Gerät besitzt als Anregungsquelle ein Gleichstromplasma (= DCP) und in der Optik ein EchelleGitter mit Prisma extrem hoher Auflösung. Im Gegensatz zum Originalgerät wurde auch hier mit elektronischen Mass FlowReglern für die einzelnen Ar-Gasströme $(2 \times$ Anode, $1 \times$ Kathode und $1 \times$ Aerosolträgergas) gearbeitet. Zur Reduzierung des Probenbedarfs von $4 \mathrm{ml} / \mathrm{min}$ auf $0,9 \mathrm{ml} / \mathrm{min}$ wurde hier ebenfalls eine peristaltische Pumpe (Gilson-Minipuls 2) eingebaut. Die Zeitdauer für eine Analyse (3 Wiederholungen und Multielementprogramm mit sequentieller Abarbeitung) beträgt für die Bestimmung von $\mathrm{Al}, \mathrm{K}, \mathrm{Li}$ und $\mathrm{Na}$ etwa 1,5 min (Integrationszeiten $0,5-2 \mathrm{~s}$ ). Auch hier wurde mit adäquater Untergrundkorrektur gearbeitet. Die Messungen erfolgten auf den in Tabelle 3 angegebenen Wellenlängen.

Tab. 3. Verwendete Wellenlängen am DCP-Spektrometer.

\begin{tabular}{ll}
\hline Element & Wellenlänge \\
\hline $\mathrm{Al}$ & 396,15 \\
$\mathrm{~K}$ & 766,49 \\
$\mathrm{Li}$ & 670,78 \\
$\mathrm{Na}$ & 568,82 \\
\hline
\end{tabular}

Bei beiden Techniken (ICP und DCP) wurde die genaue Lage der Untergrundkorrektur durch Aufnahme eines Spektrums einer realen Urinprobe ermittelt. Die Auswertung der Messungen wurde in allen Fällen über die Peak-Höhenbestimmung (15) durchgeführt. Die Kalibrierung der Geräte erfolgte jeweils unter Matrixangleichung $(\mathrm{Ca}, \mathrm{K}, \mathrm{Mg}, \mathrm{Na}, \mathrm{P})$ mit wäßrigen Standardlösungen (Merck-„Titrisole") in den geeigneten Konzentrationsbereichen. Kontrollmèssungen mittels Standard-Addition für alle Elemente ergaben keine Abweichungen gegenüber den über Standardkurven ermittelten Konzentrationen, so daß prinzipiell auf die zeitaufwendige Standard-Additionsmethode verzichtet werden kann.

\section{Voltammetrie}

Nachfolgend aufgeführte Apparate und Bedingungen wurden für die Messungen verwendet:

$\begin{array}{lll}\text { Polarograph: } & \text { PAR 264 } & \text { (Cd, Pb) } \\ & \text { PAR 384 } & \text { (Co, Ni) } \\ \text { Elektrode: } & \text { SMDE 303 } & \\ \text { Mode: } & \text { HMDE, DPASV } \\ \text { Anreicherungspotential: } & -0,90 \mathrm{~V} & \text { (Cd, Pb) } \\ & -0,70 \mathrm{~V} & \text { (Co, Ni) } \\ \text { Endpotential: } & -0,45 \mathrm{~V} & \text { (Cd, Pb) } \\ & -1,20 \mathrm{~V} & (\mathrm{Co}, \mathrm{Ni}) \\ \text { Spülzeit: } & 240 \mathrm{~s} & \\ \text { Anreicherungszeit: } & 180 \mathrm{~s} & (\mathrm{Cd}, \mathrm{Pb}) \\ & 300 \mathrm{~s} & (\mathrm{Co}, \mathrm{Ni})\end{array}$

$\begin{array}{lcl}\text { Puls-Höhe: } & 50 \mathrm{mV} & \\ \text { Scan-Rate: } & 5 \mathrm{mV} / \mathrm{s} & (\mathrm{Cd}, \mathrm{Pb}) \\ & 2 \mathrm{mV} / \mathrm{s} & (\mathrm{Co}, \mathrm{Ni}) \\ \text { pH der Lösung: } & 2,0 & (\mathrm{Cd}, \mathrm{Pb}) \\ & 9,0 & (\mathrm{Co}, \mathrm{Ni}- \\ & & \begin{array}{l}\text { Dimethyl- } \\ \text { glyoxim- }\end{array} \\ & & \text { Komplex })\end{array}$

Die normalen Kunststoff- oder Glaszellen der Elektroden wurden gegen solche aus hochreinem Quarz getauscht, um die Blindwerte zu reduzieren.

\section{Ergebnisse und Diskussion}

Die Tabellen 4 und 5 zeigen die bei diesen Untersuchungen erhaltenen Ergebnisse. Sie sind dargestellt in Konzentrationen (Tab.4) und in Ausscheidung/ $24 \mathrm{~h}$ (Tab. 5). Die Fehler bei der Analyse (bezogen auf 3 Wiederholungen) liegen im Bereich von $1-2 \%$ mit Ausnahme aller Elemente mit sehr niedrigen Konzentrationen (Bereich $\mu \mathrm{g} / \mathrm{l}$ ) wie $\mathrm{Al}, \mathrm{Cd}, \mathrm{Co}, \mathrm{Cu}, \mathrm{Fe}$, $\mathrm{Ni}, \mathrm{Pb}, \mathrm{Ti}$. Bei diesen Elementen können sie bis zu $\pm 15-20 \%$ ansteigen. Die voltammetrischen Ergebnisse in Tabelle.4 stammen aus der Untersuchung einer Mischprobe von 50 24-h-Sammelurin-Proben (Durchschnittswert).

Der Vergleich mit den in der Literatur $(2,16)$ angeführten "Referenzwerten“ zeigt eine relativ gute Übereinstimmung bei den Matrixelementen (Mineralelemente). Anders ist die Situation erfahrungsgemäß bei den Spurenelementen. Hier ist durch die Verfeinerung der Analysentechniken einschließlich der Probennahme -lagerung und -aufbereitung eine eindeutige Tendenz zu niedrigeren Werten festzustellen (17, 18), was sich auch bei den hier vorliegenden Ergebnissen für praktisch alle Spurenelemente widerspiegelt. Die Unterschiede in der Anzahl der untersuchten Urine für die verschiedenen Elemente ergeben sich aus der Tatsache, daß nicht immer alle Elemente in einer Probe bestimmt wurden. Wie bereits erwähnt, wurden die Konzentrationen von $\mathrm{Cd}, \mathrm{Co}, \mathrm{Ni}$ und $\mathrm{Pb}$ aus einer Mischprobe aus 50 Proben (des jeweils 24h-Sammelurin) bestimmt, so daß hier eine Bereichsangabe nicht gegeben werden kann.

In diesem Zusammenhang ist auch von Interesse, da $\beta$ bei der statistischen Auswertung der Ergebnisse keine signifikanten Unterschiede zwischen den männlichen und weiblichen Testpersonen festgestellt werden konnten.

Die Abbildungen 1-15 zeigen die Häufigkeitsverteilungen der Urinmengen $/ 24 \mathrm{~h}$, die Kreatininausscheidung/24h $(19,20,21)$ und die Ausscheidungen $/ 24 \mathrm{~h}$ für die untersuchten Elemente mit Ausnahme von $\mathrm{Al}$, $\mathrm{Cd}, \mathrm{Co}, \mathrm{Ni}, \mathrm{Pb}, \mathrm{Ti}$, da es dort auf Grund der äußerst niedrigen Werte nicht sinnvoll erschien. 
Tab. 4. Konzentrationen von Mineral- und Spurenelementen im menschlichen Urin. (24-h-Sammelurin, Erwachsene).

\begin{tabular}{|c|c|c|c|c|c|c|c|c|c|c|}
\hline \multirow[t]{2}{*}{$\begin{array}{l}\text { Ele- } \\
\text { ment }\end{array}$} & \multirow[t]{2}{*}{$\begin{array}{l}\text { Bestimmungs- } \\
\text { Methode }\end{array}$} & \multirow{2}{*}{$\begin{array}{l}\text { Anzahl } \\
\mathrm{n}\end{array}$} & \multirow{2}{*}{$\begin{array}{l}\text { Mittel- } \\
\text { wert } \\
\mu \mathrm{g} / \mathrm{l}\end{array}$} & \multirow[t]{2}{*}{ SD } & \multirow{2}{*}{$\begin{array}{l}\text { Mittel- } \\
\text { wert } \\
\text { mg/l }\end{array}$} & \multirow[t]{2}{*}{ SD } & \multicolumn{2}{|c|}{ Bereich } & \multirow{2}{*}{$\begin{array}{l}\text { Bereich } \\
\text { mg/l }\end{array}$} & \multirow{2}{*}{$\begin{array}{l}\text { Litera } \\
\text { tur } \\
\mu \mathrm{g} / \mathrm{l} \\
\mathrm{mg} / \mathrm{l}^{*}\end{array}$} \\
\hline & & & & & & & $\mu \mathrm{g} / 1$ & & & \\
\hline $\mathrm{Al}$ & $\mathrm{DCP}$ & 50 & 15 & 12 & - & - & & -30 & $.1-$ & 300 \\
\hline $\mathrm{Ba}$ & ICP/seq. & 25 & 4,5 & 4,2 & - & - & & $2-12,7$ & - & 10 \\
\hline $\mathrm{Ca}$ & $\mathrm{ICP} / \mathrm{sim}$ & 105 & - & - & 195 & 82 & & - & $70-390$ & $280^{*}$ \\
\hline $\mathrm{Cd}$ & Voltammetrie & 50 & 0,8 & - & - & - & & - & $=$ & 20 \\
\hline Co & Voltammetrie & 50 & 0,45 & - & - & - & & - & - & - \\
\hline $\mathrm{Cu}$ & $\begin{array}{l}\mathrm{ICP} / \mathrm{sim} \\
\mathrm{DCP}\end{array}$ & 105 & 12 & 3,4 & - & - & 2 & -20 & - & 350 \\
\hline $\mathrm{Fe}$ & $\begin{array}{l}\text { ICP/sim. } \\
\text { DCP }\end{array}$ & 105 & 11 & 14 & - & - & 2 & -70 & - & 250 \\
\hline K & $\mathrm{DCP}$ & 54 & - & - & 1982 & 640 & & - & $660-3270$ & $2300^{*}$ \\
\hline $\mathrm{Li}$ & $\mathrm{DCP}$ & 40 & 58 & 50 & - & - & 10 & -300 & $=$ & 300 \\
\hline $\mathrm{Mg}$ & $\begin{array}{l}\mathrm{ICP} / \mathrm{sim} . \\
\mathrm{DCP}\end{array}$ & 105 & - & - & 135 & 79 & & - & $40-426$ & $175^{*}$ \\
\hline $\mathrm{Na}$ & $\begin{array}{l}\text { ICP/seq. } \\
\mathrm{DCP}\end{array}$ & 40 & - & - & 3584 & 1370 & & - & $1590=7022$ & $2840^{*}$ \\
\hline $\mathrm{Ni}$ & Voltammetrie & 50 & 1,5 & $=$ & - & $=$ & & $=$ & - & 100 \\
\hline$P$ & $\begin{array}{l}\text { ICP/sim. } \\
\text { DCP }\end{array}$ & 105 & - & - & 525 & 288 & & - & $220-1070$ & $360^{*}$ \\
\hline $\mathrm{Pb}$ & Voltammetrie & 50 & 21,5 & - & - & - & & $=$ & - & 50 \\
\hline $\mathrm{Sr}$ & ICP/seq. & 25 & 240 & 181 & - & - & 27 & -798 & - & 400 \\
\hline $\mathrm{Ti}$ & $\mathrm{ICP} / \mathrm{seq}$. & 25 & 1 & - & - & - & & - & $=$ & 100 \\
\hline $\mathrm{Zn}$ & $\mathrm{ICP} / \mathrm{sim}$. & 105 & 450 & 240 & $=$ & - & 180 & -850 & - & 450 \\
\hline Kreati & in nach Jaffé (20) & 50 & - & - & $1,3^{+}$ & $0,45^{+}$ & - & & $0,44-2,41+$ & $=$ \\
\hline
\end{tabular}

$+\mathrm{g} / \mathrm{l}$

Tab. 5. Ausscheidung/24-h von Mineral- und Spurenelementen durch den menschlichen Urin (24-h-Sammelurin, Erwachsene).

\begin{tabular}{|c|c|c|c|c|c|c|c|c|c|c|}
\hline \multirow[t]{2}{*}{$\begin{array}{l}\text { Ele- } \\
\text { ment }\end{array}$} & \multirow{2}{*}{$\begin{array}{l}\text { Be- } \\
\text { stim- } \\
\text { mungs- } \\
\text { me- } \\
\text { thode }\end{array}$} & \multirow{2}{*}{$\begin{array}{l}\text { An- } \\
\text { zahl } \\
\text { n }\end{array}$} & \multirow{2}{*}{$\begin{array}{l}\text { Mit- } \\
\text { tel- } \\
\text { wert } \\
\mu \mathrm{g} / 24 \mathrm{~h}\end{array}$} & \multirow[t]{2}{*}{$\mathrm{SD}$} & \multirow{2}{*}{$\begin{array}{l}\text { Mit- } \\
\text { tel- } \\
\text { wert } \\
\mathrm{mg} / 24 \mathrm{~h}\end{array}$} & \multirow[t]{2}{*}{$\mathrm{SD}$} & \multirow{2}{*}{$\begin{array}{l}\text { Bereich } \\
\mu \mathrm{g} / 24 \mathrm{~h}\end{array}$} & \multirow{2}{*}{$\begin{array}{l}\text { Bereich } \\
\mathrm{mg} / 24 \mathrm{~h}\end{array}$} & \multirow{2}{*}{$\begin{array}{l}\text { Lit. (2) } \\
-\therefore \\
\mu \mathrm{g} / 24 \mathrm{~h} \text {, } \\
\mathrm{mg} / 24 \mathrm{~h}^{*} \\
\text { Bereich }\end{array}$} & \multirow{2}{*}{$\begin{array}{l}\text { Lit. (16) } \\
\text { Mittel } \\
\text { wert } \\
\mu \mathrm{g} / 24 \mathrm{~h} \text {, } \\
\mathrm{mg} / 24 \mathrm{~h}^{*}\end{array}$} \\
\hline & & & & & & & & & & \\
\hline $\mathrm{Al}$ & $\mathrm{DCP}$ & 50 & 20 & 16 & - & - & $7-40$ & - & $=$ & - \\
\hline $\mathrm{Ba}$ & ICP/seq. & 25 & 6 & 6 & - & - & $0,2-\quad 21$ & - & - & - \\
\hline $\mathrm{Ca}$ & $\begin{array}{l}\mathrm{ICP} / \mathrm{sim} \\
\mathrm{DCP}\end{array}$ & 105 & - & - & 261 & 149 & - & $65-510$ & $130-330^{*}$ & $230^{*}$ \\
\hline $\mathrm{Cu}$ & $\begin{array}{l}\text { ICP/sim. } \\
\text { DCP }\end{array}$ & 105 & 17 & 5 & - & - & $3-35$ & - & $3,6-32$ & 18 \\
\hline $\mathrm{Fe}$ & $\begin{array}{l}\text { ICP/sim. } \\
\text { DCP }\end{array}$ & 105 & 15 & 20 & - & - & $3-98$ & - & $40-150$ & 100 \\
\hline $\mathrm{K}$ & $\mathrm{DCP}$ & 54 & - & - & 3230 & 701 & - & $1070-5330$ & $1368-3128 *$ & $2033^{*}$ \\
\hline $\mathrm{Li}$ & DCP & 40 & 65 & 52 & - & - & $19-402$ & - & - & - \\
\hline $\mathrm{Mg}$ & $\begin{array}{l}\mathrm{ICP} / \mathrm{sim} . \\
\mathrm{DCP}\end{array}$ & 105 & - & - & 208 & 118 & - & $64-593$ & $60-200^{*}$ & $118^{*}$ \\
\hline $\mathrm{Na}$ & $\begin{array}{l}\text { ICP/seq. } \\
\text { DCP }\end{array}$ & 40 & - & - & 4180 & 1526 & - & $1580-9050$ & $2760-5060^{*}$ & $3506^{*}$ \\
\hline $\mathrm{P}$ & $\begin{array}{l}\text { ICP/sim. } \\
\text { DCP }\end{array}$ & 105 & - & - & 684 & 313 & - & $217-1470$ & $800-2000^{*}$ & - \\
\hline $\mathrm{Sr}$ & ICP/seq. & 25 & 332 & 318 & - & - & $24-1170$ & - & $=$ & - \\
\hline $\mathrm{Zn}$ & $\begin{array}{l}\text { ICP/sim. } \\
\text { DCP }\end{array}$ & 105 & 570 & 280 & - & - & $150-1400$ & - & $138-722$ & 407 \\
\hline \multicolumn{2}{|c|}{$\begin{array}{l}\text { Kreatinin nach } \\
\text { Jaffé (20) }\end{array}$} & 54 & - & - & 1900 & 558 & - & $810-3300$ & - & $\begin{array}{l}1-1,5 \\
g / 24 h(19)\end{array}$ \\
\hline
\end{tabular}




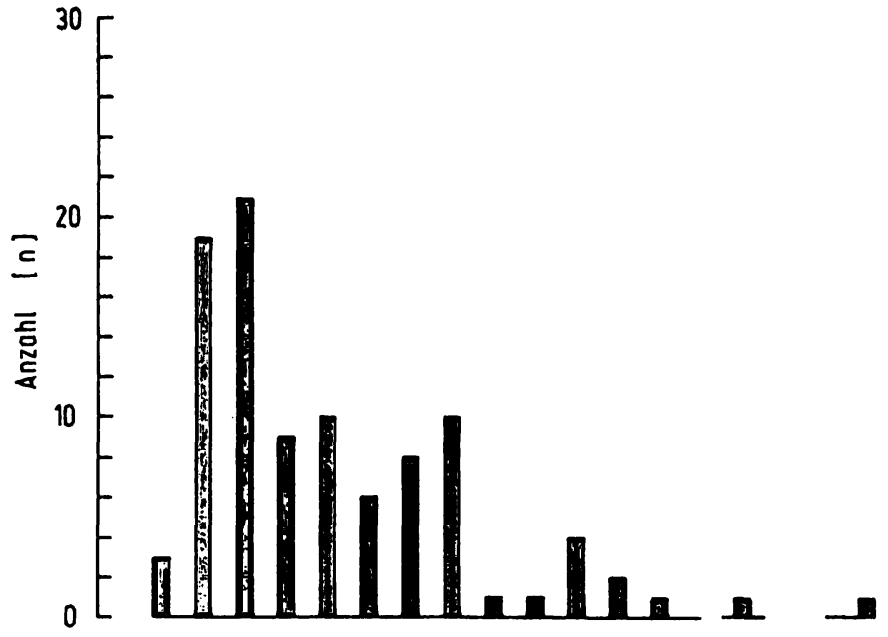

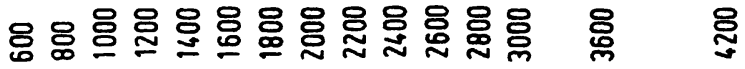
́́一́

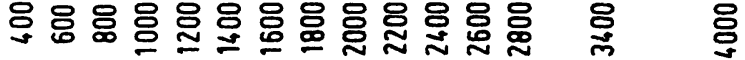
Harnzeitvölumen $[\mathrm{ml} / 26 \mathrm{~h}]$

Abb. 1. Häufigkeitsverteilung der Harnzeitvolumina.

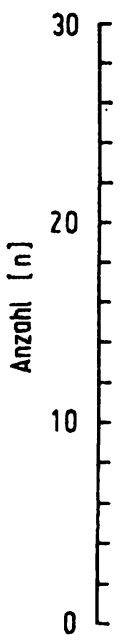

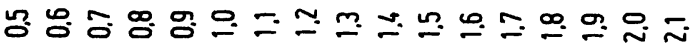

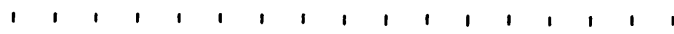
궁 웅응 둥 Kreatinin $[g / 1]$

Abb. 2. Häufigkeitsverteilung der gemessenen Kreatinin-Konzentration (in $\mathrm{g} / \mathrm{l}$ Urin).

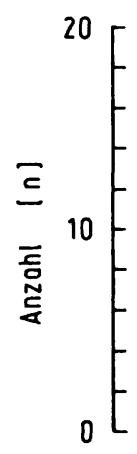

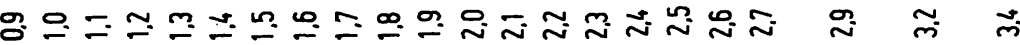

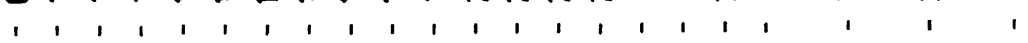

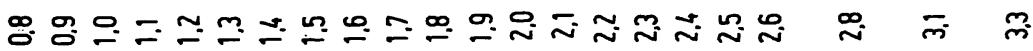
Kreạtinin-Ausscheidung $[\mathrm{g} / 24 \mathrm{~h}]$

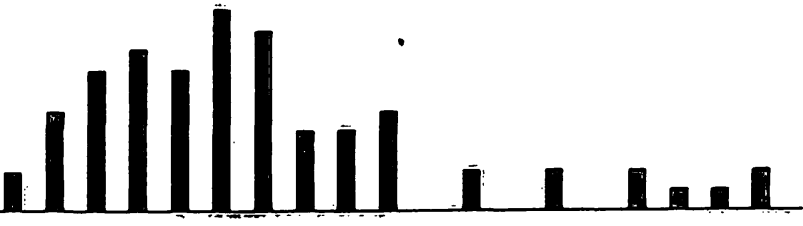

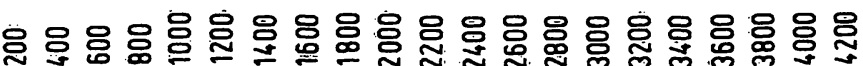

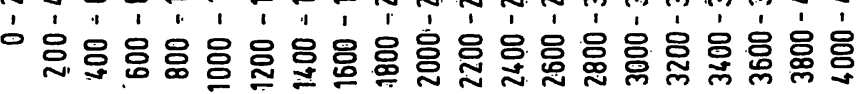

$$
\text { B - Ausscheidung }\{\mu g / 24 h\}
$$

Abb. 3. Häufigkeitsverteilung der gemessenen Kreatinin-Ausscheidung im Urin $/ 24 \mathrm{~h}$.

Abb. 4. Häufigkeitsverteilung der Bor-Aușșcheidung durch den Urin. 


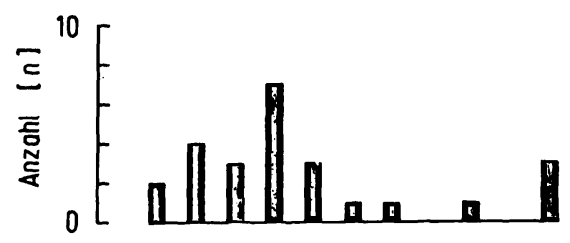

$\sim+\infty \infty$ 은ำ유

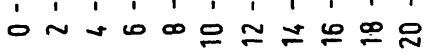

Bo-Ausscheidung $(\mu g / 24 \mathrm{~h})$

Abb. 5. Häufigkeitsverteilung der Barium-Ausscheidung durch den Urin.

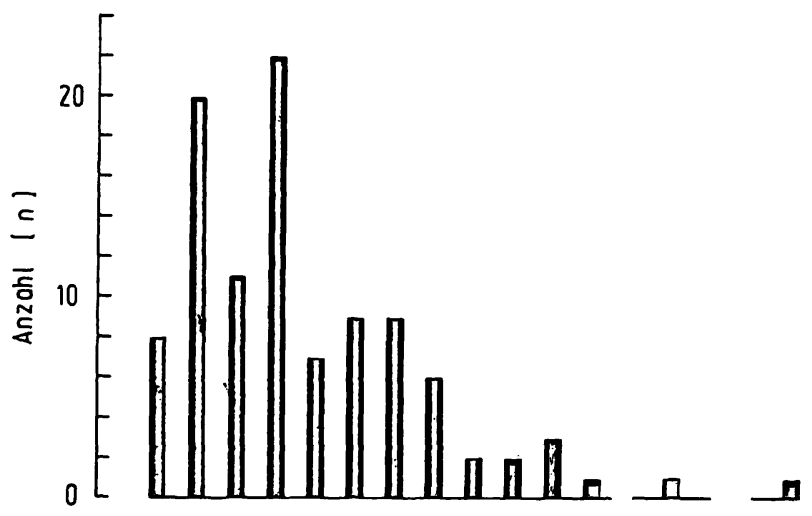

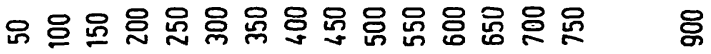

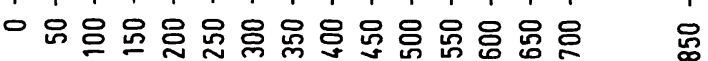

Ca-Ausscheidung $[\mathrm{mg} / 24 \mathrm{~h}]$

Abb.6. Häufigkeitsverteilung der Calcium-Ausscheidung durch den Urin.

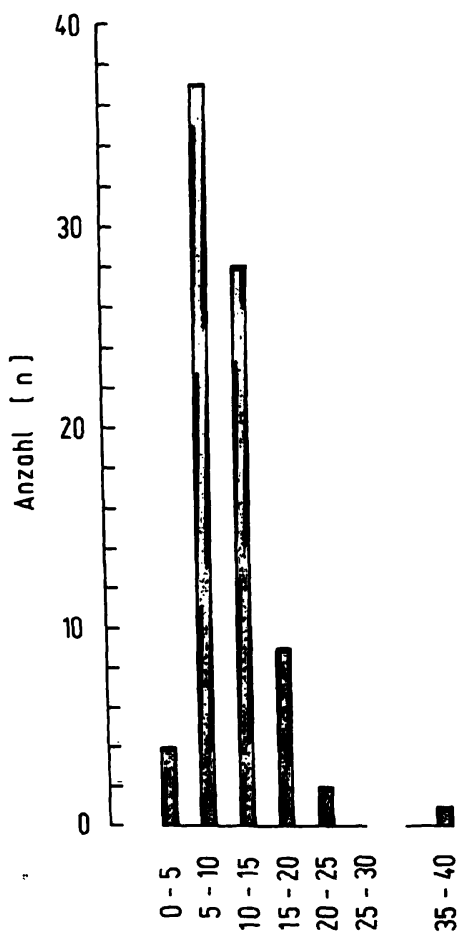

Cu-Ausscheidung $[\mu g / 24 \mathrm{~h}]$

Abb. 7. Häufigkeitsverteilung dẹ Kupfer-Ausscheidung durch den Urin.

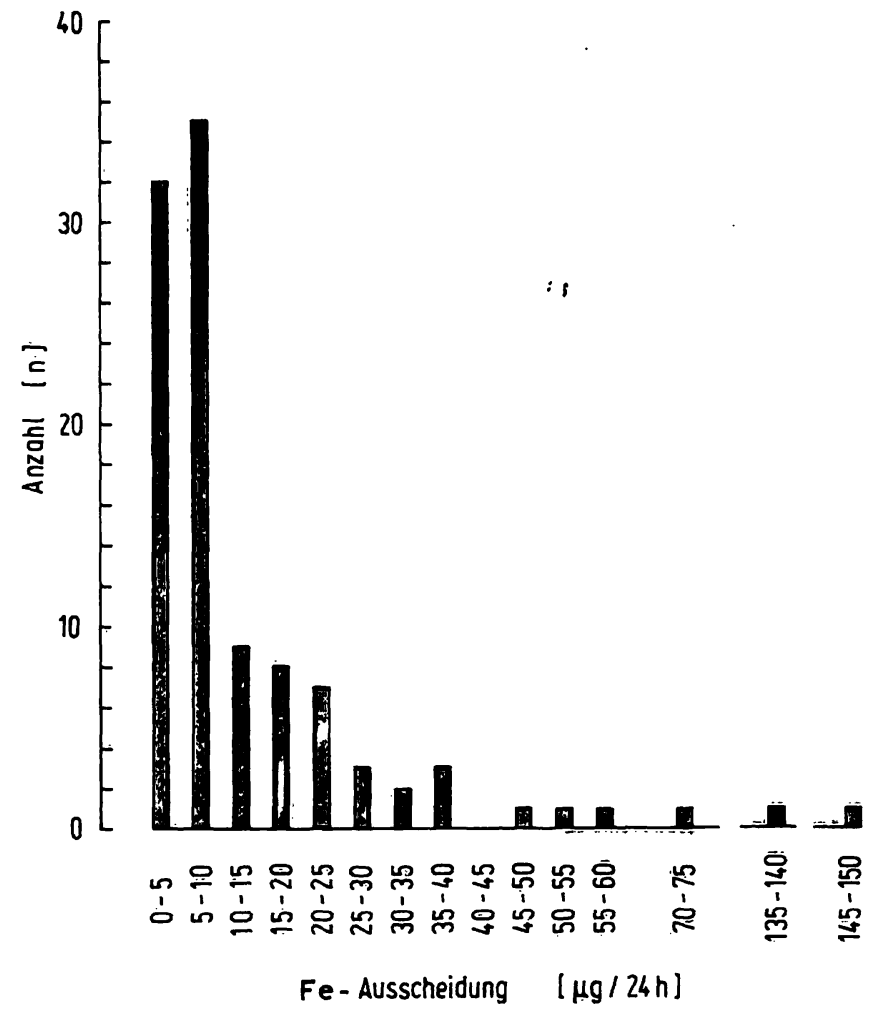

Abb. 8. Häufigkeitsverteilung der Eisen-Ausscheidung durch den Urin.

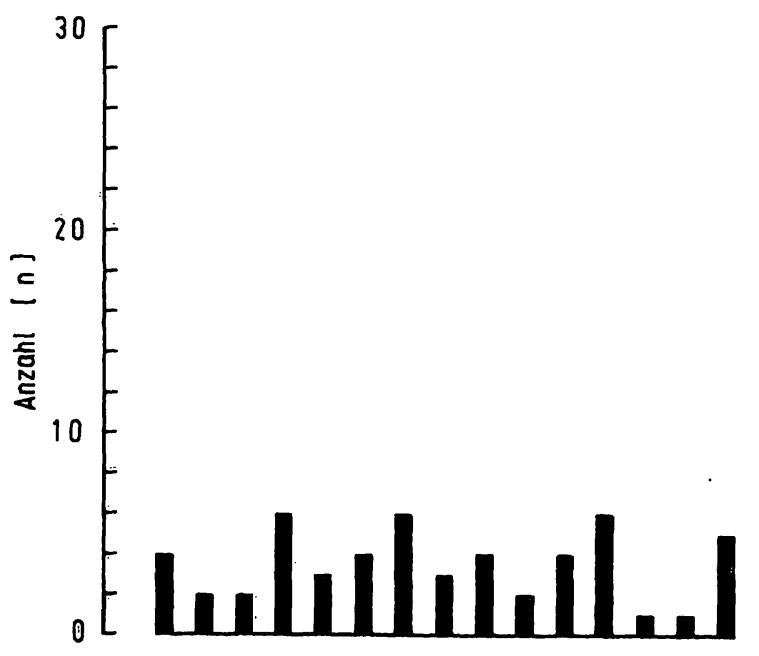

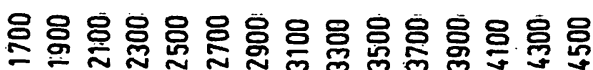

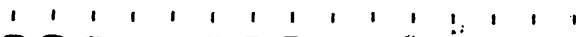

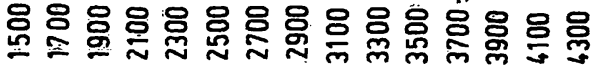

$$
K \text {-Ausscheidung }[\mathrm{mg} / 24 \mathrm{~h}]
$$

Ảbb. 9. Häufigkeitsverteilung der Kalium-Ausscheidung durch den Urin. 


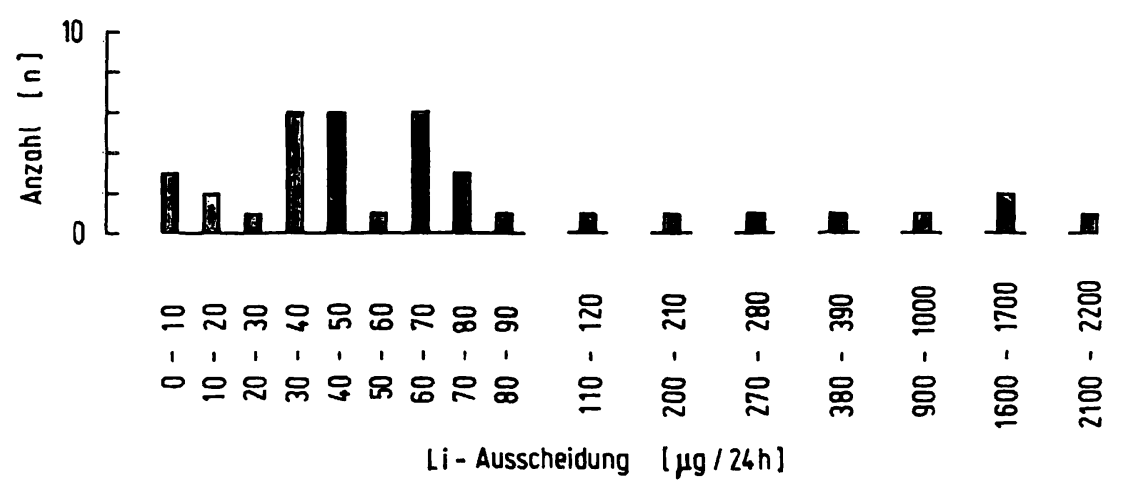

Abb. 10. Häufigkeitsverteilung der Lithium-Ausscheidung durch den Urin.

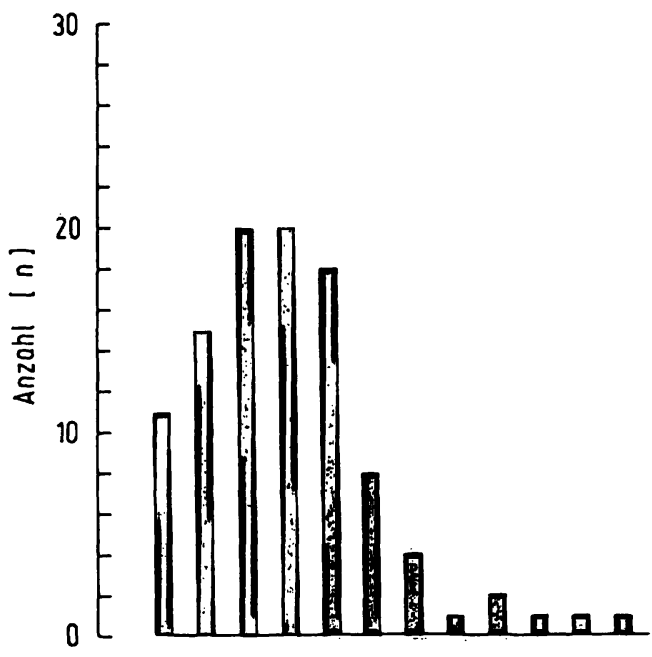

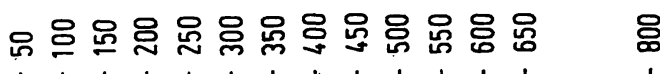

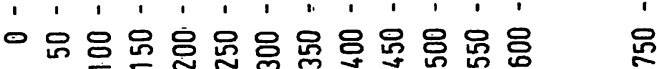

$$
\mathrm{Mg} \text {-Ausscheidung [mg/24h] }
$$

Abb.11. Häufigkeitsverteilung der Magnesium-Ausscheidung durch den Urin.

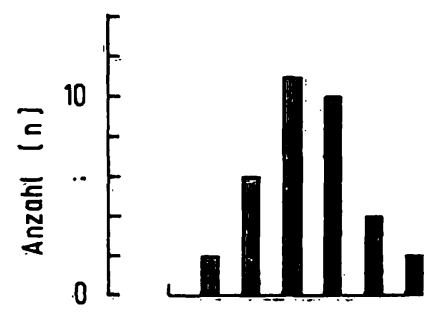

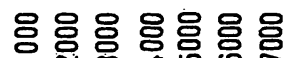
1 i 11,1

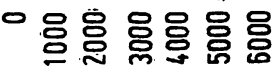

No - Ausscheidung $[\mathrm{mg} / 24 \mathrm{~h}$ ]

Abb. 12. Häufigkeitsverteilung der Natrium-Ausscheidung durch den Urin.

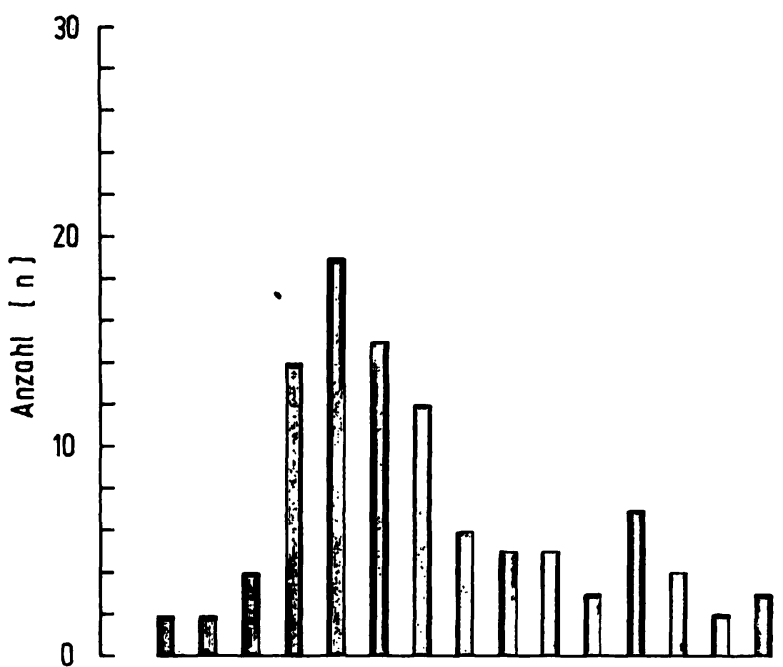

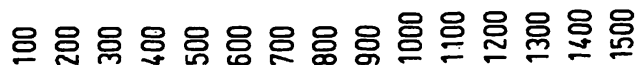
$1,1,1,1,1,1,1,1$

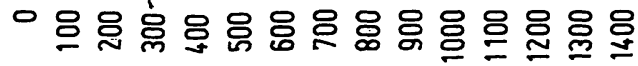

$$
P_{\text {gesamt }} \text { - Ausscheidung }[\mathrm{mg} / 24 \mathrm{~h}]
$$

Abb. 13. Häufigkeitsverteilung der Phosphor-Ausscheidung durch den Urin.

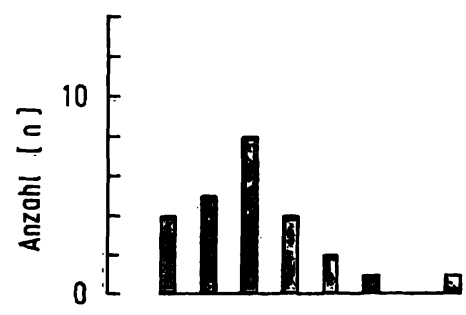

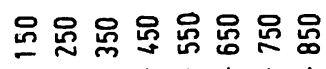

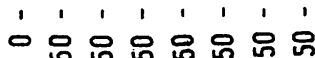

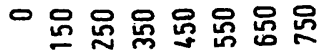

Sr-Ausscheidung $[\mu g / 24 h]$

Abb. 14. Häufigkeitsverteilung der Strontium-Ausscheidung durch den Urin. 


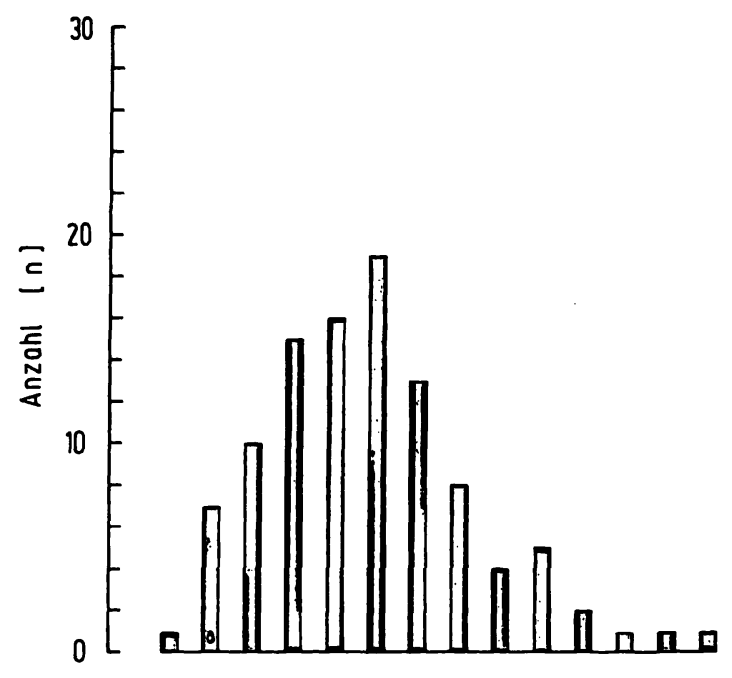

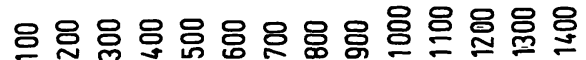
$1,1,1,1,1,1,1$

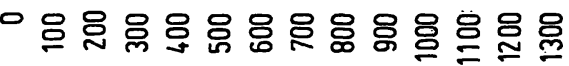

$$
\text { Zn-Ausscheidung [ }[\mu / 24 \mathrm{~h}]
$$

Abb. 15. Häufigkeitsverteilung der Zink-Ausscheidung durch den Urin.

Wegen der in der Praxis oft üblichen Bezugnahme von gemessenen Mineral- und Spurenelementkonzentrationen auf den Kreatinin-Wert bei willkürlich genommener Urinprobe (d.h. nicht 24-h-Sammelurin) wurden die Korrelationen Kreatinin/Element $\mathrm{X}$ gerechnet. Dabei ergab sich nur für Kreatinin/P (Abb. 16) und Kreatinin/Zn (Abb. 16) ein Korrelationskoeffizient $>0.9$. Alle anderen Elemente zeigen Koeffizienten $<0.8$.

In diesem Zusammenhang soll auch auf die Notwendigkeit hingewiesen werden, zur aussagekräftigen Urin-Analytik nur 24-h-Sammelurin zu verwenden, da die Mineral- und Spurenelementkonzentrationen starken tageszeitlichen Schwankungen unterworfen sind, die sehr stark von den Trink- und Eßgewohnheiten des jeweiligen Probanden und natürlich auch von dessen Nierenfunktion abhängig sind. Abbildung 18 zeigt am Beispiel eines Probanden eine zeitliche Abhängigkeit der Konzentration einiger Elemente.

Tabelle 6 zeigt schließlich einige arbeitsmedizinische Daten für Ba- und Ti-belastete Personen. Dabei handelt es sich bei den Probanden 1-5 um Schweißer, die mit dem neuen „Open Air"-Verfahren arbeiten, bei dem der Fülldraht lösliches Barium enthält. Damit finden sich auch lösliche Ba-Verbindungen im Schweißrauch und können somit vom Schweißer aufgenommen werden. Bei dem Probanden 6 handelt es sich um einen Arbeiter, der Titan-Dämpfen ausgesetzt war.

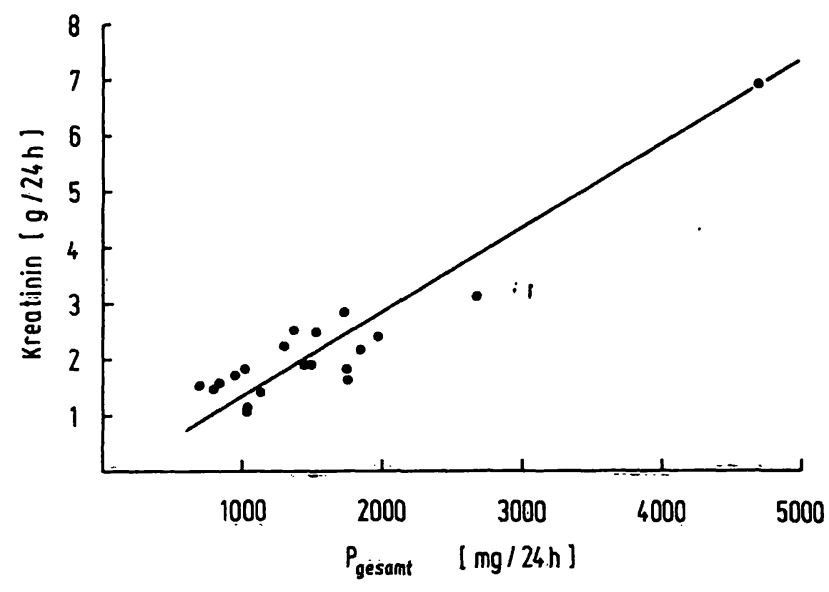

Abb. 16. Korrelation zwischen Kreatinin- und Phosphor̈Ausscheidung.

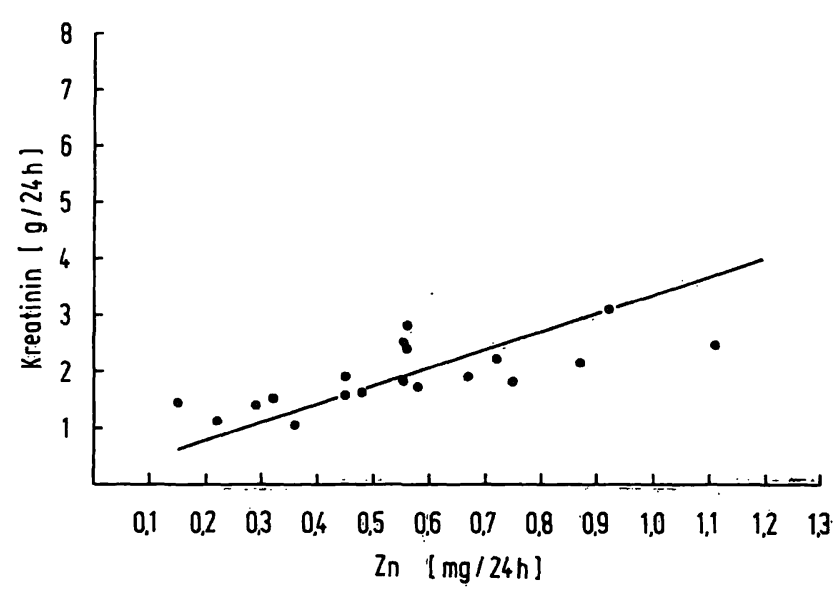

Abb. 17. Korrelation zwischen Kreatinin- und ZinkAusscheidung.

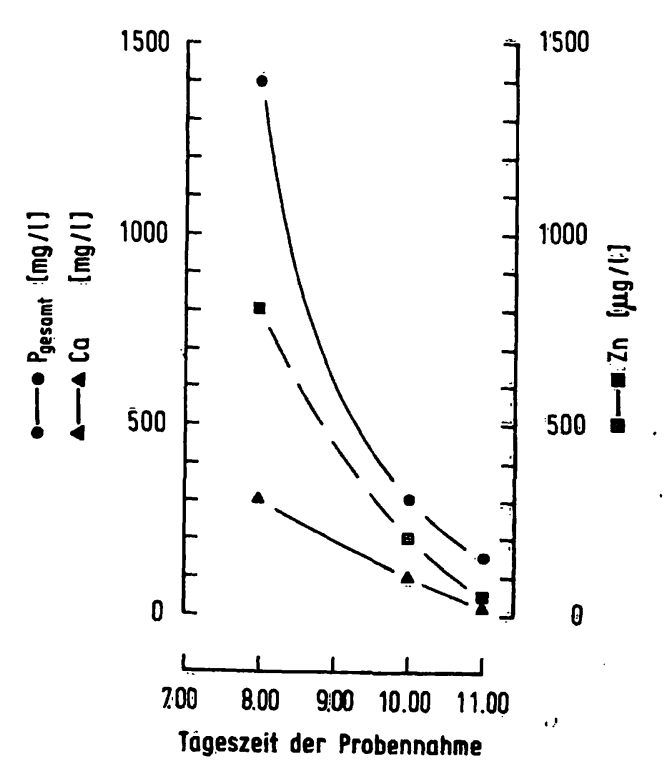

Abb. 18. Verlauf der Konzentration von $\mathrm{Ca}(\Delta=\Delta)$, $\mathrm{P}$ (-) und Żn ( von der Probennahme. $\because$

J. Clin. Chem. Clín. Biochem. / Vol. 23, $198 \dot{5}$ / No. 5 
Tab. 6. Beispiele für Ba- bzw. Ti-Belastungen am Arbeitsplatz (in $\mu \mathrm{g} / 1$ Urin).

\begin{tabular}{llll}
\hline $\begin{array}{l}\text { Proben/ } \\
\text { Element }\end{array}$ & $\mathrm{Ba}$ & $\mathrm{Ti}$ & $\begin{array}{l}\text { "Normal“- } \\
\text { werte }\end{array}$ \\
\hline 1 & 122 & - & 4,5 \\
2 & 26 & - & - \\
3 & 62 & - & - \\
4 & 185 & - & - \\
5 & 198 & - & - \\
6 & - & 13 & 1 \\
\hline
\end{tabular}

Die hier vorliegenden Ergebnisse zeigen unserer Meinung nach deutlich, daß sowohl ICP-als auch DCPEmissionsspektroskopie sehr gut für die Bestimmung einiger wichtiger Mineral- und Spurenelemente im menschlichen Urin herangezogen werden können (Tab. 7). Dies gilt insbesondere für die medizinische, einschließlich der arbeitsmedizinischen, Diagnostik und Therapiekontrolle (z. B. zeigen Morbus-WilsonPatienten eine $\mathrm{Cu}$-Ausscheidung von $>100 \mu \mathrm{g} / 24 \mathrm{~h}$ ), da sowohl der physiologische Bereich als auch ein eventueller Krankheits- oder Belastungsfall auf Grund der hohen Nachweisstärke dieser Verfahren deutlich unterschieden werden können. Die Grenzen der Verfahren werden natürlich für die physiologischen Bereiche von Elementen wie $\mathrm{Cd}, \mathrm{Co}, \mathrm{Ni}$ und $\mathrm{Pb}$ erreicht, so daß in diẹsen Fällen immer andere, geeignetere Techniken wie z. B. die Voltammetrie oder die flammenlose Atomabsorptionsspektroskopie ein-

\section{Literatur}

1. Anke, M. (1984) Trace Element Analytical Chemistry in Medicine and Biology, Vol. III, pp. 421-465. (Brätter, P. \& Schramel, P., eds.) Verlag W. de Gruyter, Berlin.

2. Documenta Geigy, Wissenschaftliche Tabellen (1969) S. R. Geigy S. A., Bạsel.

3. Iyengar, G. V., Kollmer, W. E. \& Bowen, H. J. M. (1978) The Elemental Composition of Human Tissues and Body Fluids, Verlag Chemie, Weilheim.

4. Beršin, Th. (1963) Biochemie der Mineral- und Spurenelemente, Akadèmische Vèrlagsgesellschaft, Frankfurt/Main.

5. Schramel, P., Wolf, A., Seif, R. \& Klose, B.-J. (1980) Fresenius Z. Anal. Chemie, 302, 62-64.

6. Hasse, S. \& Schramel, P. (1983) Mikrochim. Äcta III, $449-455$.

7. Schramel, P., Wolf, A. \& Klose, B.-J. (1980) J. Clin. Chem. Cilin. Biochem. 18, 591-593.

8. Schramel, P. \& Klose, B.-J. (1981) Fresenius Z. Anal. Chem. $307,26-30$.

9. Schramel, P. \& Xu, L. Q. (1982) ICP Inf. Newsl. 7, $429-440$.

10. Schramel, P. \& Xu, L. Q. (1983) Fresenius Z. Anal. Chem. $314,671-677$.

11. Schramel, P. (1984) Fresenius Z. Anal. Chem. 319, $229-239$

12. Schramel, P. \& Ovcar-Pavlu, J. (1979) Fresenius Z. Anal. Chem. 298, 28-31.
Tab. 7. Nachweisgrenzen der Elemente bei ICP- und DCPSpektroskopie (gemessen in Urin-Proben).

\begin{tabular}{lccl}
\hline $\begin{array}{l}\text { Ele- } \\
\text { ment }\end{array}$ & ICP & DCP & $\begin{array}{l}\text { Voltammetrie } \\
(\mu \mathrm{g} / \mathrm{l})\end{array}$ \\
\hline $\mathrm{Al}$ & 50 & $(\mu \mathrm{g} / \mathrm{l})$ & 5 \\
$\mathrm{Ba}$ & 0,4 & 0,3 & \\
$\mathrm{Ca}$ & 0,4 & 0,7 & \\
$\mathrm{Cu}$ & 5 & 5 & \\
$\mathrm{Fe}$ & 5 & 5 & \\
$\mathrm{~K}$ & 400 & 20 & \\
$\mathrm{Li}$ & 20 & 2 & \\
$\mathrm{Mg}$ & 0,2 & 0,2 & \\
$\mathrm{Na}$ & 10 & 3 & \\
$\mathrm{P}$ & 100 & 100 & \\
$\mathrm{Sr}$ & 0,1 & 0,3 & \\
$\mathrm{Ti}$ & 1 & 3 & \\
$\mathrm{Zn}$ & 5 & 10 & \\
$\mathrm{Cd}$ & & & 0,1 \\
$\mathrm{Co}$ & & & 0,2 \\
$\mathrm{Ni}$ & & & 1 \\
$\mathrm{~Pb}$ & & & 2 \\
\hline
\end{tabular}

gesetzt werden müssen. Insgesamt erscheint aber die Plasma-Emissionsspektroskopie für dieses Anwendungsgebiet besser geeignet $\mathrm{zu}$ sein als die in der Praxis übliche Flammen-Emissions- oder -Absorptionsspektroskopie. Allerdings sind die Geräte noch relativ teuer und erfordern auch höher qualifiziertes Bedienungspersonal, so daß der Einsatz im klinischchemischen Labor noch etwas problematisch erscheint.

13. Schramel, P., Fischer, R., Wolf, A. \& Hasse, S. (1981) ICP Inf. Newsl. 8, 401-408.

14. Boorn, A. W., Bosshart, R. E., Karlinski, T. J., Babis, J. \& Zander, A. T. (1983) Plasma Line 4, 4-13.

15. Schramel, P., Klose, B.-J. \& Hasse, S. (1982) Fresenius Z. Anal. Chemie 310, 209-216.

16. Panteliedes, C., Boenigk, H. E. \& Janke, W. (1975) Infusionstherapie 2, 377-386.

17. Cornelis, R. \& Versieck, J. (1980) Trace Element Analytical Chemistry in Medicine and Biology, Vol. I, pp. 587-600, (Brätter, P. \& Schramel, P., eds.) Verlag W. de Gruyter, Berlin.

18. Stoeppler, M. (1984) Trace Element Analytical Chemistry in Medicine and Biology, Vol. III, pp. 539-537 (Brätter, P. \& Schramel, P., eds.) Verlag W. de Gruyter, Berlin.

19. Seelig, H. P. \& Wüst, H. (1969) Ärztl. Lab. 15, 34-38.

20. Schirmeister, J., Willman, H. \& Kiefer, H. (1964) Dtsch. Med. Wochenschr. 87, 1018-1024.

21. Sarre, H. (1959) Nierenkrankheiten, Georg Thieme Verlag, Stuttgart.

Dr. Peter Schramel Gesellschaft für Strahlenund Umweltforschung $\mathrm{mbH}$ Physikalisch-Technische Abteilung Arbeitsgruppe "Spurenelementanalytik" Ingolstädter Landstraße 1 D-8042 Oberschleißheim 
